\title{
Concentração de cálcio no dialisato e hipercalcemia na DRC
}

Dialysate calcium concentration and hypercalcemia in CKD

Autores:

Leandro Junior Lucca

Rosélia Ribeiro dos

Santos Lobão

Cristina Karohl
1 Concentração de Ca no dialisato

1.1 A concentração de Ca no dialisato $(\mathrm{Cad})$ deve ser individualizada (Opinião).

1.2 A Cad deve estar preferencialmente entre 2,5 e $3,0 \mathrm{mEq} / \mathrm{L}$.

1.2.1 Nos pacientes com níveis séricos de paratormônio $(\mathrm{PTH})$ inferiores a $150 \mathrm{pg} / \mathrm{mL}$, a Cad deve ser preferencialmente de $2,5 \mathrm{mEq} / \mathrm{L}$ (Opinião).

1.2.2 Nos pacientes com níveis séricos de PTH acima de $500 \mathrm{pg} / \mathrm{mL}$ e sem hipercalcemia, a Cad deve ser preferencialmente de $3,0 \mathrm{mEq} / \mathrm{L}$ (Opinião).

1.3 A Cad de 3,5 mEq/L deve ser utilizada preferencialmente quando houver necessidade de balanço positivo de $\mathrm{Ca}$ (Opinião).

1.3.1 A Cad de 3,5 mEq/L deve ser sempre utilizada após a PTx até o término do período de "fome óssea" (Opinião).

2 Hipercalcemia na doença renal crônica (DRC)

Pacientes com DRC estágios III e IV

2.1 Os níveis séricos de Ca devem ser mantidos dentro da faixa normal para o método (Evidência).

Pacientes com DRC estágio $V$

2.2 Os níveis séricos de Ca devem ser mantidos dentro da faixa normal para o método (Opinião).

2.2.1 Se utilizar o Ca iônico (Cai), este deve ser mantido dentro da faixa de normalidade, não excedendo o limite de 5\% abaixo do valor superior do método empregado (Opinião).

2.3 Hipercalcemia associada a PTH menor que $300 \mathrm{pg} / \mathrm{mL}$, seguir as seguintes recomendações:

2.3.1 Utilizar dialisato com concentração de Ca de 2,5 mEq/L por 2 meses (Opinião).

2.3.2 Interromper o tratamento com vitamina $\mathrm{D}_{2}$ ou $\mathrm{D}_{3}$ (Opinião).

2.3.3 Mudar o quelante de $\mathrm{P}$ à base de Ca por um quelante sem Ca e sem alumínio (Evidência).

2.3.4 Depois de seguidas as Diretrizes 2.3.1 a 2.3.3, se o Ca estiver dentro dos valores recomendados, as condutas devem ser mantidas (Opinião).

2.3.4.1 Se o $\mathrm{Ca}$ ainda permanecer acima dos valores recomendados, manter as Diretrizes 2.3.1 a 2.3.3 por 2 meses ou mais até que o Ca esteja dentro dos valores recomendados, desde que os níveis de PTH estejam abaixo de $300 \mathrm{pg} / \mathrm{mL}$ (Opinião).

2.3.4.2 Excluir outras causas de hipercalcemia não relacionadas com as anormalidades do metabolismo mineral e ósseo da doença renal crônica (Opinião).

2.4 Hipercalcemia associada a PTH maior ou igual a $300 \mathrm{pg} / \mathrm{mL}$, seguir as seguintes recomendações:

2.4.1 Interromper o tratamento com vitamina $\mathrm{D}_{2}$ ou $\mathrm{D}_{3}$ (Opinião). 
2.4.2 Mudar o quelante de $\mathrm{P}$ à base de Ca por um quelante sem Ca e sem alumínio (Opinião).

2.4.3 Depois de seguidas as Diretrizes 2.4.1 e 2.4.2, se o Ca estiver dentro dos valores recomendados, as condutas devem ser mantidas e devese reiniciar o tratamento com vitamina $\mathrm{D}_{2}$ ou $\mathrm{D}_{3}$ (Opinião).

2.4.3.1 Se o Ca estiver acima dos valores recomendados, manter as Diretrizes 2.4.1 e 2.4 .2 e utilizar dialisato com concentração de $\mathrm{Ca}$ de 2,5 $\mathrm{mEq} / \mathrm{L}$ por 2 meses, de acordo com as Diretrizes da Concentração de Ca no dialisato. Se após 2 meses, os níveis de Ca estiverem acima dos valores recomendados, indicar paratireoidectomia de acordo com as Diretrizes de Paratireoidectomia (Evidência).

\section{RACIONAL}

A concentração ideal de Ca no dialisato é motivo de controvérsia. ${ }^{1-3}$ Há uma tendência de tentar generalizar uma concentração ideal para todos os pacientes em diálise, mas isso deve ser reavaliado principalmente porque as características clínicas e necessidades dos pacientes não são as mesmas. No entanto, parece claro que o tratamento adequado da Cad é um fator importante e deveria ser considerado parte do tratamento dos distúrbios do metabolismo mineral e ósseo da DRC. ${ }^{4}$

$\mathrm{Na}$ realidade, desde a década de 1960, época em que a HD foi introduzida como terapia de substituição da função renal, as recomendações da Cad foram principalmente baseadas em opinião de especialistas e de acordo com a situação clínica de cada época, mais do que em evidências. Na década de 1960, as maiores preocupações eram controlar os níveis séricos de $\mathrm{P}$ e de Ca com o objetivo de prevenir doença óssea e calcificação metastática em pacientes mantidos em diálise regular. ${ }^{5,6}$ A Cad de 2,5 mEq/L foi arbitrariamente estabelecida, visando ao balanço neutro e ao uso de quelante de $\mathrm{P}$ à base de alumínio associado à suplementação oral de Ca. Essas eram as principais medidas adotadas na época. Posteriormente, observou-se a necessidade de maiores cargas de $\mathrm{Ca}$ no dialisato, uma vez que os pacientes desenvolviam hipocalcemia e hiperparatireoidismo secundário (HPS). A partir dessas observações, foi proposto aumentar o Ca no dialisato para 3,5 mEq/L com o objetivo de fornecer carga maior de $\mathrm{Ca}$ e suprimir a secreção de PTH. $^{7}$ Com o reconhecimento da toxicidade do alumínio, os quelantes com Ca passaram a ser utilizados para a maioria dos pacientes. ${ }^{8}$ No entanto, hipercalcemia começou a ser observada, especialmente quando se associava ao calcitriol. Consequentemente, no final da década de 1980 e início da década de 1990, grande parte dos nefrologistas retornou ao uso de Cad de 2,5 $\mathrm{mEq} / \mathrm{L}$. Essa mudança foi suportada por outros estudos que mostraram que o uso combinado de carbonato de $\mathrm{Ca}$, calcitriol e Ca no dialisato de $2,5 \mathrm{mEq} / \mathrm{L}$ era efetivo para tratar o HPS. ${ }^{9-11}$

Mais recentemente, sobrecarga de $\mathrm{Ca}$, mesmo na ausência de hipercalcemia, foi associada a maior risco de calcificação vascular, doença óssea de baixa remodelação e mortalidade, aumentando a preocupação com o ganho de Ca pelos pacientes em diálise, seja do quelante ou do dialisato. ${ }^{12,13}$ Além disso, a associação de maior risco de mortalidade em pacientes com níveis mais elevados de Ca observado em estudo observacional reforçou esta ideia. ${ }^{14}$ Young e cols., analisando os resultados do primeiro Dialysis Outcomes and Practice Patterns Study (DOPPS), encontraram uma elevação de $13 \%$ no risco de mortalidade geral para cada aumento de $1 \mathrm{mEq} / \mathrm{L}$ de Ca no dialisato. ${ }^{15}$ Essa preocupação com a sobrecarga de $\mathrm{Ca}$ foi um dos pontos determinantes para a recomendação de uma concentração de $2,5 \mathrm{mEq} / \mathrm{L}$ de Ca no dialisato pelo K/DOQI para a maioria dos pacientes, exceto em situações específicas, quando este pode ser mais alto ou baixo. ${ }^{1} \mathrm{Na}$ prática clínica, o primeiro DOPPS mostrou que apenas $40 \%$ dos pacientes estavam em uso de uma Cad recomendada pelo K/ DOQI. O uso de uma concentração de $2,5 \mathrm{mEq} / \mathrm{L}$ foi principalmente observada nos Estados Unidos com cerca de $64 \%$ dos pacientes dialisatos nessa solução. $\mathrm{Na}$ Europa e no Japão, apenas $24 \%$ e $20 \%$ estavam em uso de Cad de 2,5 mEq/L, respectivamente. ${ }^{15}$ No mais recente guia para o tratamento dos distúrbios do metabolismo mineral e ósseo da DRC foi sugerido o uso de Cad de 2,5 ou 3,0 mEq/L. ${ }^{3}$

Apesar das poucas evidências sobre a concentração ideal de Ca no dialisato, algumas considerações devem ser observadas. A decisão sobre a concentração de Ca no dialisato deve levar em consideração a necessidade individual do balanço de Ca durante a diálise. Recentemente, demontrou-se uma grande variabilidade individual na transferência de $\mathrm{Ca} \mathrm{du}$ rante a diálise independente da Cad. Tanto o gradiente de Ca entre o sangue e o dialisato e a taxa de remodelação óssea estimada pelos níveis séricos de PTH foram fatores determinantes da transferência de Ca. ${ }^{16}$ Dessa forma, o tipo de doença óssea, avaliado por marcadores bioquímicos ou pela biópsia óssea, além da presença ou não de calcificação 
extraóssea deveriam ser considerados na escolha da concentração de Cad. Em geral, Cad de 3,5 mEq/L associa-se com balanço positivo e concentrações inferiores a 2,5 $\mathrm{mEq} / \mathrm{L}$ com balanço negativo de $\mathrm{Ca}$ durante a diálise. ${ }^{17-19}$ Naqueles pacientes com níveis séricos de PTH menores que $150 \mathrm{pg} / \mathrm{mL}$, a concentração de $\mathrm{Ca}$ recomendada no dialisato é de 2,5 $\mathrm{mEq} / \mathrm{L}$, com o objetivo de estimular a remodelação óssea, visto que a prevalência de DOA é elevada em pacientes com esses níveis de $\mathrm{PTH} .{ }^{20,21}$ No entanto, Cad iguais ou menores a 2,5 mEq/L deveriam ser usadas com cautela em pacientes com níveis séricos de PTH elevados. Excessiva remoção de Ca poderia agravar o HPS e causar significativa perda de massa óssea. Por outro lado, o uso de Cad de $3,5 \mathrm{mEq} / \mathrm{L}$ é associado a um balanço positivo de $\mathrm{Ca}$ em uma parcela considerável dos pacientes e deveria ser evitado em pacientes com níveis de PTH mais baixo, os quais, geralmente, apresentam doença óssea adinâmica, menor capacidade de tamponar Ca e maior risco para calcificação vascular.

O Ca desempenha importantes papéis fisiológicos no organismo humano. No osso, participa da integridade estrutural do esqueleto. No fluido extracelular e no citosol, a concentração de íons Ca, geralmente mantida constante, é criticamente importante na manutenção e no controle de inúmeros processos bioquímicos. $^{22}$

Considerando-se um nível sérico de Ca total de $10,0 \mathrm{mg} / \mathrm{dL}, 45 \%$ representam a porção ligada à albumina, sendo os $55 \%$ restantes representados pelo Cai, importante para funções biológicas, e apenas $0,5 \mathrm{mg} / \mathrm{dL}$ do Ca total encontra-se complexado com outros íons como citrato, bicarbonato e fosfato. É importante mencionar que, nos pacientes com DRC estágios III, IV e V, devemos utilizar preferencialmente as dosagens do Cai ou o Ca total corrigido para o nível sérico da albumina. ${ }^{23}$ Para o cálculo de Ca total corrigido, deve-se utilizar a seguinte fórmula: $\mathrm{Ca}$ total corrigido $=\mathrm{Ca}$ total medido $+[(4-$ al bumina ) x 0,8$]$.

Nos pacientes com DRC, níveis séricos de Ca acima dos limites normais foram associados a aumento no risco de mortalidade. ${ }^{14}$ Ingestão de Ca na dieta, o tipo de quelante de $\mathrm{P}$ utilizado, o uso de vitamina $\mathrm{D}_{2}$ ou $\mathrm{D}_{3}$, uso de calcimimético, nível sérico do PTH e concentração de $\mathrm{Ca}$ no dialisato podem influenciar a concentração sérica de Ca nesses pacientes. ${ }^{15}$

A ingestão diária de Ca inclui não só o aporte alimentar, mas também o Ca proveniente do quelante de P. Atualmente, recomenda-se que a ingestão diária de $\mathrm{Ca}$, considerando aquela proveniente do quelante de $\mathrm{P}$, não exceda a $2 \mathrm{~g} /$ dia. A quantidade de Ca elementar contida no carbonato e acetato de Ca é $40 \%$ e $25 \%$ respectivamente. A vitamina D2 e D3 aumenta a absorção intestinal de $\mathrm{Ca}$ e $\mathrm{P}$ podendo causar hipercalcemia e/ou hiperfosfatemia. ${ }^{24,25}$ Importante ressaltar que, na presença de hipercalcemia não associada ao tratamento ou ao tipo de doença óssea renal, outras causas devem ser investigadas.

\section{REFERÊNCIAS}

1. National Kidney Foundation. K/DOQI clinical practice guidelines for bone metabolism and disease in chronic kidney disease. Am J Kidney Dis. 2003; 42(suppl 3):S1-S201.

2. Toussaint N, Cooney P, Kerr PG. Review of dialysate calcium concentrations in hemodialysis. Hemodialysis Int. 2006; 10:326-37.

3. Kidney Disease. Improving Global Outcomes (KDIGO) CKD-MBD Work Group. KDIGO clinical practice guideline for the diagnosis, evaluation, prevention and treatment of chronic kidney disease-mineral and bone disorder (CKD-MBD). Kidney International 2009; 76(Suppl 113):S1-S130.

4. Palmer B. Individualizing the dialysate in the hemodialysis patient. Semin Dial. 2001; 14:41-9.

5. Slatopolsky E. Recommendations for treatment of renal osteodystrophy in dialysis patients. Kidney Int. 1975; 7(suppl 2):253-5.

6. Pendras JP, Erickson RV. Hemodialysis: A successful therapy for chronic uremia. Annals Intern Med. 1966; 64:293-310.

7. Johnson WJ, Goldsmith RS, Beabout JW et al. Prevention and reversal of progressive secondary hyperparathyroidism in patients maintened by hemodialysis. Am J Med 1974; 56:827-32.

8. Slatopolsky E, Weerts C, Lopez-Hilker S et al. Calcium carbonate as a phosphate binder in patients with chronic renal failure undergoing dialysis. $\mathrm{N}$ Engl J Med. 1986; 315:157-61.

9. Slatopolsky E, Weerts C, Norwood K et al. Long term effects of calcium carbonate and $2,5 \mathrm{mEq} / \mathrm{L}$ calcium dialysate on mineral metabolism. Kidney Int. 1989; 36:897-903.

10. Sawyer N, Noonan K, Altmann P et al. High-dose calcium carbonate with stepwise reduction in dialysate calcium concentration: effective phosphate control and aluminum voidance in hemodialysis patients. Nephrol Dial Transplant. 1989; 4:105-9.

11. Van der Merwe WM, Rodger RS, Grant AC et al. Low calcium dialysate and high-dose oral calcitriol in the treatment of secondary hyperparathyroidism in hemodialysis patients. Nephrol Dial Transplant. 1990; 5:874-7.

12. Goodman WG, Goldin J, Kuizon BD et al. Coronary artery calcification in young adults with end-stage renal disease who are undergoing dialysis. $\mathrm{N}$ Engl J Med. 2000; 342:1478-83.

13. Chertow GM, Burke SK, Raggi P. Sevelamer attenuates the progression of coronary and aortic calcification in hemodialysis patients. Kidney Int. 2002; 62:245-52. 
14. Block GA, Klassen PS, Lazarus JM, Ofsthun N, Lowrie EG, Chertow GM. Mineral metabolism, mortality, and morbidity in maintenance hemodialysis. J Am Soc Nephrol. 2004; 15:2208-18.

15. Young EW, Albert JM, Satayathum S et al. Predictors and consequences of altered mineral metabolism:the Dialysis Outcomes and Practice Patterns Study. Kidney Int. 2005; 67:1179-87.

16. Karohl C, Paschoal JP, Castro MCM et al. Effects of bone remodelling on calcium mass transfer during hemodiálisis. Nephrol Dial Transplant. 2010; 25:1244-51.

17. Angiles A, Mourad G. How do we have to use the calcium in the dialysate to optimmize the management of secondary hyperparathyroidism. Nephrol Dial Transpl. 1998; 13(suppl 3):62-4.

18. Malberti F, Surian M, Minetti L. Dialysate calcium concentration decrease exacerbates secondary hyperparathyroidism in dialysis patients given calcium carbonate as a phosphate binder. J Nephrol. 1991;2:75-81.

19. Fabrizi F, Bacchini G, Di Filippo S et al. Intradialytic calcium balances with different calcium dialysate levels. Nephron. 1996; 72:530-5.
20. Barreto FC, Barreto DV, Moysés RMA, et al. K/DOQIrecommended intact PTH levels do not prevent low-turnover bone disease in hemodialysis patients. Kidney Int. 2008; 73:771-7.

21. Hamano T, Oseto S, Fujii $\mathrm{N}$ et al. Impact of lowering dialysate calcium concentration on serum boné turnover markers in hemodialysis patients. Bone 2005; 36:909-16.

22. Llach F, Bover J. Renal osteodystrophy. In: Brenner BM. Brenner \& Rector's The Kidney 6.ed. Philadelphia: WB Saunders Company; 2000, p. 2103-86.

23. Payne RB, Carver ME, Morgan DB. Interpretation of serum total calcium: effects of adjustment for albumin concentration on frequency of abnormal values and on detection of change in the individual. J Clin Pathol. 1979; 32(1):56-60.

24. Brown AJ, Coyne DW. Vitamin D analogs: new therapeutic agents for secondary hyperparathyroidism. Treat Endocrinol. 2002; 1:313-27.

25. Henley C, Colloton M, Cattley RC et al. 1,25-Dihydroxyvitamin D3 but not cinacalcet $\mathrm{HCl}$ (Sensipar/Mimpara) treatment mediates aortic calcification in a rat model of secondary hyperparathyroidism. Nephrol Dial Transplant. 2005; 20:1370-7. 